\title{
Teoria delle sostituzioni che operano su una infinità numerabile di elementi.
}

\author{
Memoria $2^{a}\left({ }^{*}\right)$ di LUIGI ONOFRI (a Bologna).
}

CAPITOLO III.

IS OM ORFISM I

\section{A) Isomorfismo oloedrico.}

59. Due gruppi o pseudogruppi $\mathfrak{A}, \mathfrak{B}$ aventi il medesimo ordine si dicono isomorf oloedrictmente quando si può stabilire fra le loro operazioni una corrispondenza biunivoca tale che al prodotto di due sostituzioni qualsiasi di $\mathfrak{A}$ corrisponda in $\mathscr{B}$ il prodotto delle corrispondenti.

Per indicare che due complessi $\mathfrak{C}, \mathfrak{D}$, appartenenti rispettivamente ad $\mathfrak{A}$ e $\mathfrak{B}$, sono formati con operazioni corrispondenti scriveremo:

$$
\mathcal{C} \cos \text {. }
$$

60. Due complessi $\mathfrak{A}, \mathfrak{B}$ isomorf oloedricamente sono entrambi gruppi, oppure sono pseudogruppi della medesima specie.

Supponiamo che in $\mathfrak{A}$ esista l'operazione identica; dalla corrispondenza

$$
1 \cos b
$$

discende :

$$
1 \cdot 1 \operatorname{cob} b \cdot b, \quad b^{2}=b, \quad b=1,
$$

onde anche in $\mathfrak{B}$ esiste l'identità.

Sia poi a una operazione di $\mathfrak{G}$ che possiede l'inversa in $\mathfrak{A}$; dalle corrispondenze:

$$
a \cos b, a^{-1} \cos b_{1}
$$

(*) Vedi Memoria $1^{\text {a }}$, "Annali di Matematica ", Serie IV, Tomo IV, Fasc. 1.2, p. 73. 
si deducono le altre:

$$
a \cdot a^{-1}=1 \cos b \cdot b_{1}, \quad b_{1}=b^{-1},
$$

e quindi l'operazione $b$ possiede l'inversa in $\mathfrak{B}$.

In particolare, se $\mathfrak{A}$ e $\mathfrak{B}$ sono rispettivamente gli pseudogruppi composti $G+G^{\prime}, \Gamma+\Gamma^{\prime}$, si ha:

$$
G \cos , \quad G^{\prime} \cos \Gamma^{\prime}
$$

61. Ad esempio, i complessi $\mathfrak{A}$ e $t^{-1} \cdot \mathfrak{A} \cdot t$ sollo isomorfi oloedricamente secondo la corrispondenza:

$$
a \cos t^{-1} \cdot a \cdot t
$$

Se $\mathfrak{G}$ é invariante per $t$, la $(0)$ definisce un isomorfismo fra $\mathfrak{A}$ e sè stesso, se invece $\mathfrak{A}$ è riducibile (ampliabile) mediante $t$, la $(o)$ definisce un isomorfismo fra $\mathfrak{Q}$ ed una sua parte propria (fra $\mathfrak{A}$ ed un complesso che lo contiene).

62. Siano $\mathfrak{C}, \mathfrak{D}$ due complessi appartenenti rispettivamente ad $\mathfrak{A}, \mathfrak{B}$ e tali che $\mathfrak{C} \cos$.

Vogliamo dimostrare che : se $\mathfrak{C} \dot{e}$ invarianle in $\mathfrak{A}, \mathfrak{D}$ è invarianle in $\mathfrak{B}$. Invero, prese due operazioni qualsiasi $b, d$ di $\mathfrak{B}, \mathfrak{D}$ e posto:

$$
a \sin b, \quad c \cos d,
$$

si ha, per l'invarianza di $\mathfrak{C}$ in $\mathfrak{A}$ :

$$
a \cdot c=c_{1} \cdot a, \quad c \cdot a=a \cdot c_{2} ;
$$

da cui, per il supposto isomorfismo:

$$
b \cdot d=d_{1} \cdot b, \quad d \cdot b=b \cdot d_{2} .
$$

In modo analogo si prova la riducibilità (l'ampliabilità) di $\mathfrak{D}$ mediante $\mathscr{B}$, ammesso che $\mathcal{C}$ sia riducibile (ampliabile) mediante $\mathfrak{A}$.

$\dot{\mathrm{E}}$ infine da notare che se $\mathfrak{C}$ è invariante massimo in $\mathfrak{A}, \mathfrak{D}$ è pure invariante massimo in $\mathfrak{B}$.

63. Le proposizioni dei precedenti n. $^{\mathrm{i}} 60,62$ dimostrano l' equivalenza dei due complessi isomorfi $\mathfrak{A}$ e $\mathfrak{B}$ rispetto al modo con cui le operazioni di ciascun complesso si compongono tra loro. E pertanto lecito di riportarsi, per lo studio di un complesso $\mathfrak{A}$, ad un complesso $\mathfrak{B}$ isomorfo ad $\mathfrak{A}$.

Ad esempio, si puó sostituire ad $\mathfrak{A}$ uno speciale complesso, detto congiunto di $\mathfrak{Q}$, formato con sostituzioni i cui elementi sono le operazioni del gruppo $\left(\mathfrak{A}, \mathfrak{A}^{-1}\right)$. 
Per effettuare in ogni caso la costruzione di questo complesso è necessario introdurre delle operazioni diverse da quelle finora considerate, e precisamente delle sostituzioni su insiemi di elementi aventi potenze diverse dal numerabile.

La definizione di queste nuove operazioni si ottiene da quella del $n .{ }^{0} 1$ sostituendo all' insieme numerabile un insieme qualunque. Tutte le proprietà e le definizioni che abbiamo dato, e che non siano inerenti alla natura delle sostituzioni, restano valide per queste nuove operazioni $\left({ }^{1}\right)$.

64. Dato un complesso $\mathfrak{A}$, formiamo il gruppo $G=\left(\mathfrak{A}, \mathfrak{A}^{-1}\right)$ e facciamo corrispondere alla sostituzione generica $g$ di $G$ quella operazione $k$ che sostituisce ad ogni $x$ di $G$ l'operazione $x \cdot g$ pure di $G$. Scriveremo:

$$
k=\left(\begin{array}{c}
x \\
x \cdot g
\end{array}\right) \text {. }
$$

Il complesso $K$ di tutte queste operazioni $h$ ha, evidentemente, la medesima potenza di $G$ ed è isomorfo a $G$ secondo la corrispondenza superiormente definita.

Infatti al prodotto $g \cdot g$, corrisponde l'operazione:

$$
k^{\prime}=\left(\begin{array}{c}
x \\
x \cdot g \cdot g_{1}
\end{array}\right)=\left(\begin{array}{c}
x \\
x \cdot g
\end{array}\right) \cdot\left(\begin{array}{c}
x \\
x \cdot g_{1}
\end{array}\right)=k \cdot k_{1} \text {. }
$$

Consideriamo ora il complesso $\mathfrak{K}$ formato con le operazioni di $K$ che corrispondono alle operazioni di $\mathfrak{A}$.

Tale complesso è isomorfo ad $\mathfrak{A}$, è della sua stessa specie, e prende il nome di. congiunto a destra di $\mathfrak{G}$.

Il congiunto a sinistra si ottiene in modo analogo moltiplicando le operazioni di $G$ a sinistra anzichè a destra, e ponendo la corrispondenza $: g \circ\left(\begin{array}{c}x \\ g^{-1} \cdot x\end{array}\right)$.

\section{B) Isomorfismo meriedrico.}

65. Dati due orruppi o pseudogruppi $\mathfrak{A}, \mathfrak{B}$ diremo che $\mathfrak{A}$ è isomor fo meriedricamenle a $\mathfrak{B}$ quando:

a) ad ogni sostiturione di $\mathfrak{A}$ ne corrisponde una ed una sola in $\mathfrak{B}$, mentre ad una di $\mathfrak{B}$ ne corrispondono diverse in $\mathfrak{A}$;

(1) Lo studio sistematico di queste sostituzioni esce dai limiti imposti alla presente Teoria: esse serviranno esclusivamente per la costruzione di complessi isomorfi a complessi dati (vedi ancho i N.i 75, 76). 
b) al prodotto di due qualsiasi sostituzioni di $\mathfrak{A}$ corrisponde il prodotto delle corrispondenti di $\mathfrak{B}$.

Il simbolo in introdotto al $n .^{\circ} 59$ servirà, anche per questo isomorfismo, ad indicare la corrispondenza fru le operazioni di $\mathfrak{E}$ e quelle di $\mathfrak{B}$.

66. Con un ragionamento analogo a quello fatto al $\mathrm{n}^{\circ} 60$, si prova per l'attuale isomorfismo che:

a) Se in $\mathfrak{A}$ figura l'operazione identica, la sua corrispondente in $\mathfrak{B}$ è pure l'identità.

B) Se a e una operazione di $\mathfrak{Q}$ che ha l'inversa in $\mathfrak{G}$, la corrispondente $\mathrm{b}$ di $\mathfrak{B}$ ha l'inversa in $\mathfrak{B}$.

Poiché alla identita in $\mathfrak{B}$ corrispondono varie operazioni di $\mathfrak{A}$, le proposizioni precedenti non sono generalmente invertibili; e cioè :

$\left.\alpha^{\prime}\right)$ l'esistenza della identità in $\mathfrak{B}$ non porta all' esistenza della identità in $\mathfrak{a}$;

$\left.\beta^{\prime}\right)$ il complesso $\mathfrak{B}$ puó contenere simultaneamente le operazioni $b, b^{-1}$ senza che le operazioni di $\mathfrak{A}$ corrispondenti a $b$ abbiano l'inversa in $\mathfrak{A}$.

Da queste considerazioni si deduce come l'isomorfismo meriedrico permetta, a differenza di quello oloedrico, la diversita di specie nei complessi corrispondenti $\mathfrak{A}, \mathfrak{B}$, e come siano solamente possibili i seguenti tipi d'isomorfismo :

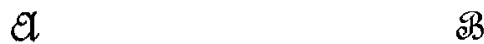

$$
\begin{array}{ll}
\text { gruppo } & \text { gruppo } \\
\text { pseudogruppo composto os } & \begin{array}{l}
\text { gruppo } \\
\text { pseudogruppo composto }
\end{array} \\
\text { pseudogruppo semplice os } & \begin{array}{l}
\text { gruppo } \\
\text { pseudogruppo composto } \\
\text { pseudogruppo semplice }
\end{array}
\end{array}
$$

Più avanti studieremo singolarmente questi vari isomorfismi e daremo degli esempi per ciascuno di essi.

67. Siano $\mathfrak{C}, \mathfrak{D}$ due complessi appartenenti rispettivamente ad $\mathfrak{A}, \mathfrak{B}$ e tali che $\mathfrak{C} \cos$.

Se $\mathcal{C}$ è invarianle in $\mathfrak{A}, \mathfrak{D}$ è invarianle in $\mathfrak{B}$ (per la dimostr. vedi n. ${ }^{\circ} 62$ ), mentre se $\mathfrak{D}$ è invariante in $\mathfrak{B}, \mathfrak{C}$ può non essere tale in $\mathfrak{A}$. 
Ciò si verifica nel seguente:

Esempio. Con il gruppo totale $A$ sugli elementi $0,1,2, \ldots, n, \ldots$ e con il ciclo :

$$
g=\left(\ldots, a_{-n}, \ldots, a_{-1}, 0,1, \ldots, n, \ldots\right)
$$

si formino gli pseudogruppi:

$$
\Gamma^{\prime \prime}=A+g \cdot A+\ldots .+g^{r} \cdot A+\ldots ., \quad \Lambda^{\prime \prime}=1+g+\ldots .+g^{r}+\ldots . .
$$

La corrispondenza $g^{r} \cdot A \cos g^{r}$ stabilisce fra $\Gamma^{\prime \prime}$ e $\Lambda^{\prime \prime}$ un isomorfismo tale che alla identita in $\Lambda^{\prime \prime}$ corrisponde il gruppo riducibile $A\left(1 .^{\circ} 50\right)$.

Se $\mathfrak{C}$ è riducibile, $\mathfrak{D}$ è invarianle o riducibile.

Infatti, se $b, d$ sono due operazioni qualsiasi di $\mathfrak{B}, \mathfrak{D}$ e se:

si ha:

$$
a \cos b, c \cos d
$$

$$
c \cdot a=a \cdot c_{1}, \text { da cui } a \cdot b=b \cdot d_{1} .
$$

In modo simile si prova che se $\mathfrak{C}$ è ampliabile, $\mathfrak{D}$ è invariante od ampliabile.

68. Studiamo dapprima l' isomorfismo meriedrico fra due gruppi $A$ e $B$.

Sia $\mathfrak{D}$ un complesso contenuto in $B$, e sia $\mathfrak{C}$ il complesso di tutte le operazioni di $A$ corrispondenti a $\mathfrak{D}$.

Mediante facilissime considerazioni si dimostra che:

o) I complessi $\mathcal{C}$, D sono entrambi gruppi, oppure sono pseudogruppi della stessa specie.

w) Se $\mathscr{D}$ è invariante in $B, \mathfrak{C} e ̀$ invariante in $A$.

Questa proposizione è degna di rilievo perchè, come si è visto al n..$^{\circ} 67$, essa puó cadere in difetto quando i complessi isomorfi sono degli pseudogruppi.

Dalle o), $\omega)$ si deduce, ponendo $\mathscr{D}=1$, che tutte le operazioni di $A$ corrispondenti all' unità in $B$ formano un gruppo invariante $K$. In conseguenza di ciò ad ogni operazione di $B$ corrispondono in $A$ tutte e sole le operazioni di un quasi-gruppo di una decomposizione di $A$ rispetto a $K$.

L'ordine del gruppo $K$ si dirà grado di meriedria.

69. Consideriamo ora $l^{\prime}$ isomorfismo fra uno pseudogruppo composto

ed un gruppo $B$.

$$
A^{\prime \prime}=A+A^{\prime}
$$

Sia $a^{\prime}$ una operazione qualunque di $A^{\prime}$ e sia $b$ la sua corrispondente in $B ; \mathrm{da}$ :

$$
a^{\prime} \cos b, a^{\prime \prime} \cos b^{-1}, a^{\prime} \cdot a^{\prime \prime} \cdot \cos 1
$$


consegue che: le operazioni di $\mathrm{A}^{\prime \prime}$ corrispondenti all'unità in $\mathrm{B}$ costituiscono uno pseudogruppo composto $\mathrm{K}^{\prime \prime}$.

Indichiamo poi con $B_{1}$ il gruppo formato con le operazioni di $B$ corrispondenti alle operazioni di $A$, e con $A_{1}^{\prime \prime}$ lo pseudogruppo composto formato con tutte le operazioni di $A^{\prime \prime}$ a cui corrispondono le operazioni di $B_{1}$.

Vogliamo dimostrare che:

a) lo pseudogr uppo $\mathrm{A}_{1}^{\prime \prime}$ è decomponibile rispetto a $\mathrm{K}^{\prime \prime}$;

b) ad ogni operazione di $\mathrm{B}_{1}$ cor"rispondono in $\mathrm{A}^{\prime \prime}$ tulte e sole le operazioni di un quasi-pseudogr'uppo di detta decomposizione.

Sia $b_{1}$ una operazione di $B_{1}$ e siano $a_{1}, a$ due operazioni di $A_{1}^{\prime \prime}, A$ corrispondenti a $b_{1}$. Da:

$$
a^{-1} \cos b_{1}^{-1}, \quad a_{1} \cos b_{1} \text { segue } a^{-1} \cdot a_{1}=k \cos 1,
$$

onde tutte le operazioni di $A_{1}^{\prime \prime}$ corrispondenti a $b_{1}$ appartengono al complesso $a \cdot K^{\prime \prime}$.

L' insieme di tutti i complessi del tipo di $a \cdot K^{\prime \prime}$ costituisce perció una decomposizione a sinistra di $A_{1}^{\prime \prime}$ rispetto a $K^{\prime \prime}$. In modo analogo si prova la decomponibilità a destra di $A_{1}^{\prime \prime}$ rispetto a $K^{\prime \prime}$.

Inoltre, poichè ciascuna di queste decomposizioni ha per potenza l'ordine di $B_{1}$, si può asserire che : gli indici di $\mathrm{K}^{\prime \prime}$ in $\mathrm{A}_{1}^{\prime \prime}$ sono eguali.

EsEmpro. Siano $A$ e $B$ due gruppi isomorfi oloedricamente e sia $\gamma$ una operazione senza periodo o con periodo infinito commutabile con tutte le operazioni di $A$.

Ponendo:

$$
\left[a, a \cdot \gamma, \ldots ., a \cdot \gamma^{*}, \ldots .\right] \cos b
$$

dove $a \cos b$ nell' isomorfismo fra $A$ e $B$, si viene a stabilire un isomorfismo meriedrico fra lo pseudogruppo:

ed il gruppo $B$.

$$
A^{\prime \prime}=A+A \cdot \gamma+\ldots+A \cdot \gamma^{r}+\ldots
$$

70. Siano $A^{\prime \prime}=A+A^{\prime}$ e $B^{\prime \prime}=B+B^{\prime}$ due pseudogruppi composti isomorfi meriedricamente.

Le operazioni di $A^{\prime \prime}$.corrispondenti alle operazioni di $B$ costituiscono un gruppo od uno pseudogruppo composto $\mathfrak{A}$; ed il gruppo $A$, corrispondendo ad un sottogruppo di $B$, deve essere contenuto in $\mathfrak{Q}$.

Si ha pertanto, se $\mathfrak{Q}$ è un gruppo, $\mathfrak{Q}=A$. 
Se $\mathfrak{A}$ è uno pseudogruppo composto, l' isomorfismo fra $\mathfrak{A}$ e $B$ è sicuramente meriedrico $\left(\mathrm{n}^{\circ}{ }^{\circ} 69\right)$, mentre se $\mathfrak{Q}=A$ il suddetto isomorfismo può essere anche oloedrico (vedi esempi al $11 .^{\circ} 74$ ).

Sia $A_{1}^{\prime}$ lo pseudogruppo semplice formato con le operazioni di $A^{\prime \prime}$ che corrispondono a quelle di $B^{\prime}$.

$\mathrm{L}^{\prime}$ isomorfismo che intercede fra $A_{i}^{\prime}$ e $B^{\prime}$ è del tipo di quelli che verramno studiati al $n 0^{\circ} 73$; esso deve risultare necessariamente meriedrico se si vuole che tale sia l' isomorfismo fra $A^{\prime \prime}$ e $B^{\prime \prime}$.

Invero, se fra $A_{\uparrow}^{\prime}$ e $B^{\prime}$ vi fosse isomorfismo oloedrico, alla identitá in $B^{\prime \prime}$ corrisponderebbe la sola identita in $A^{\prime \prime}$ e perciò ad ogni operazione di $B^{\prime \prime}$ corrisponderebbe una sola operazione di $A^{\prime \prime}$.

71. Studiamo ora gli isomorfismi fra uno pseudogruppo semplice $A^{\prime}$ ed un gruppo $B$, oppure fra il suddetto pseadogruppo ed un altro pseudogruppo composto o semplice.

Esaminiamo dapprima $l^{\prime}$ isomorfismo fra $A^{\prime}$ e $B$.

Le operazioni di $A^{\prime}$ che hanno per corrispondente l'identità in $B$ costituiscono uno pseudogruppo semplice $K^{\prime}$.

Inoltre, dalle corrispondenze:

$$
K^{\prime} \cos 1, a \cos b, K^{\prime} \cdot a \cos b
$$

si deduce che ad ogni operazione $b$ di $B$ corrispondono infinite operazioni di $A^{\prime}$.

$\dot{\mathrm{E}}$ importante notare che il complesso $K^{\prime} \cdot a$, non contenendo $a$, è formato soltanto con una parte. dell' insieme delle operazioni di $A^{\prime}$ che corrispondono a $b$.

EsEMPIO. I complessi :

$$
A^{\prime}=\left[a, a^{2}, \ldots, a^{r}, \ldots .\right], \quad B=[1, b] \quad\left(b^{2}=1\right)
$$

sono isomorfi secondo le corrispondenze:

$$
\left[a^{2}, a^{4}, \ldots . ., a^{2 n}, \ldots .\right] \propto 1, \quad\left[a, a^{3}, \ldots ., a^{2 n-4}, \ldots .\right] \cos b .
$$

72. Passiamo all' isomorfismo tra uno pseudogruppo semplice $A^{\prime}$ ed uno composto $B^{\prime \prime}=B+B^{\prime}$.

All' unitá in $B^{\prime \prime}$ corrisponde uno pseudogruppo semplice $K^{\prime}$ di $A^{\prime}$, e conseguentemente ad ogni operazione di $B^{\prime \prime}$ corrispondono infinite operazioni di $A^{\prime}$.

I complessi $C^{\prime}$ e $C_{1}^{\prime}$, formati con le operazioni di $A^{\prime}$ che corrispondono rispettivamente a $B$ ed a $B^{\prime}$, sono due pseudogruppi semplici. 
Ecco un esempio di questo isomorfismo :

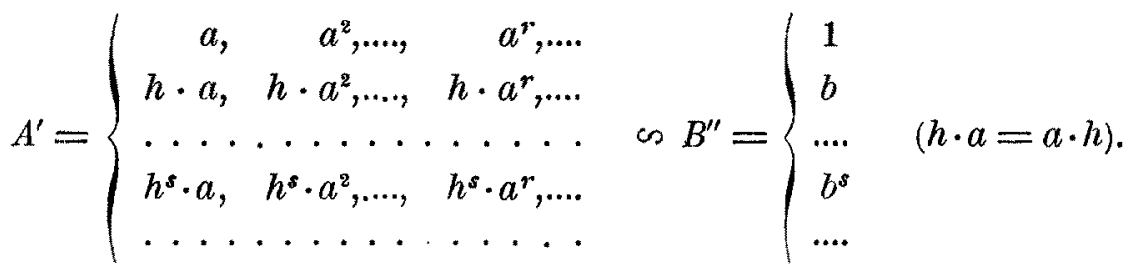

73. Resta da esaminare l' isomorfismo fra due pseudogruppi semplici $A^{\prime}$ e $B^{\prime}$.

Negli isomorfismi finora studiati tra due complessi $\mathfrak{A}, \mathfrak{B}$ (a parte quello del $\mathrm{n}^{\circ} 70$ che, come abbiamo detto, dipende dal presente isomorfismo) ad ogni operazione di $\mathfrak{B}$ corrispondono in $\mathfrak{A}$ operazioni in numero infinito oppure in egual numero finito.

Nell' attuale isomorfismo puó invece darsi che il numero delle operazioni di $A^{\prime}$ corrispondenti alle operazioni di $B^{\prime}$ varî da operazione ad operazione.

EsEMPIO I. Sia :

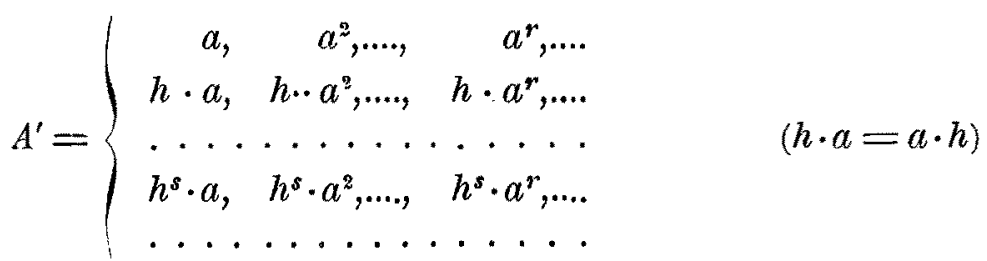

e

$$
B^{\prime}=\left[b, b^{2}, \ldots, b^{s}, \ldots\right]
$$

Ponendo la corrispondenza :

$$
\left[h^{m-1} \cdot a, h^{m-2} \cdot a^{2}, \ldots ., h^{m-n} \cdot a^{n}, \ldots, a^{m}\right] \backsim b^{m},
$$

si viene a stabilire fra $A^{\prime}$ e $B^{\prime}$ un isomorfismo tale che alla operazione $b^{m}$ di $B^{\prime}$ corrispondono in $A^{\prime}, m$ operazioni.

EsEmpIo II. Sia $A_{1}^{\prime}$ lo pseudogruppo che si ottiene togliendo dal precedente $A^{\prime}$ le operazioni $h \cdot a, h^{2} \cdot a, \ldots, h^{s} \cdot a, \ldots$.

Le corrispondenze:

$$
a \cos b, \quad\left[a^{r}, h \cdot a^{r}, \ldots ., h^{s} \cdot a^{r}, \ldots\right] \cos b^{r} \quad(r>1)
$$

definiscono un isomorfismo fra $A_{i}^{\prime}$ e $B^{\prime}$ in cui a $b$ corrisponde la sola $a$ e ad ogni altra potenza di $b$ corrispondono infinite operazioni. 
74. Torniamo al caso studiato al $\mathrm{n} .^{\circ} \mathbf{7 0}$.

$L^{\prime}$ isomorfismo fra $A_{1}^{\prime}$ e $B^{\prime}$ può essere di uno qualunque dej tipi or ora accennati; perciò le operazioni di $A^{\prime \prime}$, corrispondenti ad ogni operazione di $B^{\prime \prime}$, possono variare in numero da operazione ad operazione.

$\mathrm{Si}$ ottengono facilmente degli esempi di questo isomorfismo aggiungendo l' identità ai complessi costruiti al $n .^{\circ} 73$, ed unendo alle corrispondenze là poste la 1 cos.

In questi esempi si ha $\mathfrak{Q}=A$, mentre nel caso seguente:

si ha:

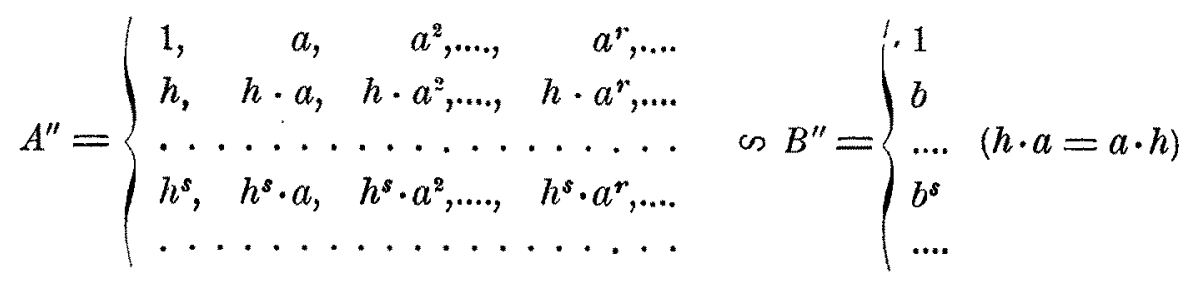

$$
\mathfrak{Q}=\left[1, a, a^{2}, \ldots ., a^{r}, \ldots .\right]
$$

75. Al $1 .^{\circ} 63$ abbiamo iutrodotto delle sostituzioni su insiemi non numerabili di elementi e ci siamo serviti di esse per costruire dei complessi isomorfi oloedricamente a dati complessi di sostituzioni:

Vogliamo ora costruire, dato un gruppo o pseudogruppo $\mathfrak{A}$, un complesso $\mathfrak{K}$ di queste operazioni isomorfo meriedricamente ad $\mathfrak{Q}$.

Sia $\Sigma(H \cdot g)$ una decomposizione del gruppo $G=\left(\mathfrak{A}, \mathfrak{Q}^{-1}\right)$ rispetto ad un suo sottogruppo qualsiasi $H$.

Alla operazione generica $\gamma$ di $G$ facciamo corrispondere quella operazione $k$ che sostituisce ad ogni quasi-gruppo $\mathcal{Q}$ il quasi-gruppo $Q \cdot \gamma$. Scriveremo:

$$
k=\left(\begin{array}{c}
\mathcal{Q} \\
\mathbf{Q} \cdot \gamma
\end{array}\right)
$$

Poichè al prodotto $\gamma \cdot \gamma_{1}$ corrisponde l'operazione :

$$
\left(\begin{array}{c}
Q \\
\mathcal{Q} \cdot \gamma_{\gamma_{1}}
\end{array}\right)=\left(\begin{array}{c}
\mathcal{Q} \\
\mathcal{Q} \cdot \gamma
\end{array}\right) \cdot\left(\begin{array}{c}
\mathcal{Q} \\
\mathcal{Q} \cdot \gamma_{1}
\end{array}\right)
$$

si può asserire che $G$ è isomorfo al gruppo $K$ formato con tutte le operazioni $k$. Vediamo quali sono le operazioni di $G$ corrispondenti all' unita in $K$.

Se $\delta$ è una di queste operazioni, deve aversi per un generico $\mathcal{Q}=H \cdot g$ :

$$
\mathcal{Q} \cdot \delta=\mathcal{Q}, \quad H \cdot g \cdot \delta=H \cdot g, \quad \delta=g^{-1} \cdot h \cdot g .
$$


L'operazione $\delta$ è dunque comune a tutti i trasformati di $H$ mediante $G$ e quindi fa parte del loro sottogruppo comune $\Delta$.

Inversamente, se $\delta$ appartiene a $\Delta$ e se $\mathcal{Q}=H \cdot g$ è un quasi-gruppo generico, si ha:

$$
\mathrm{Q} \cdot \bar{\delta}=H \cdot g \cdot \delta=H \cdot g \cdot g^{-1} \cdot h \cdot g=H \cdot g=\mathrm{Q},
$$

cioè a $\delta$ corrisponde l'identità in $K$.

Concludendo: l'isomorfismo che intercede fra $\mathrm{G}$ e $\mathrm{K}$ ha per grado di meriedria l'ordine di $\Delta$.

Consideriamo ora il complesso $\mathscr{O}$ formato con le operazioni di $K$ che corrispondono alle operazioni di $\mathfrak{G}$.

Il complesso $\mathfrak{H}$ è isomorfo ad $\mathfrak{E}$ e, secondo la sua specie, prende il nome di gruppo o pseudogruppo complementare a destra di $\mathfrak{A}$ rispello ad $\mathrm{H}$.

Scriveremo:

$$
\mathfrak{o}=\frac{\mathfrak{Q}}{H}
$$

76. Partendo da una decomposizione a sinistra, anzichè da una a destra, facendo corrispondere a $\gamma$ l'operazione $\left(\begin{array}{c}\mathcal{Q} \\ \gamma^{-1} \cdot \mathcal{Q}\end{array}\right)$, ed operando per tutto il resto in modo analogo, si ottiene un complesso $\mathfrak{H}_{1}$ isomorfo ad $\mathfrak{A}$ e detto complemenlare a sinistra.

I complessi $\mathfrak{H}$ e $\mathfrak{J K}_{1}$ sono oloedricamente isomorfi.

77. Se il complesso $\mathfrak{Q}$ non è un gruppo, vi è dubbio circa la specie di $\frac{\mathfrak{Q}}{H}$ : essa dipende anche dal gruppo $H$ che si è scelto.

Affinche $\frac{\mathfrak{Q}}{\mathrm{H}}$ sia un gruppo occorre e basta che ogni quasi-gruppo di una decomposizione $\mathbf{\Sigma}\left(\Delta \cdot g^{\prime}\right)$ di Grispelto a $\Delta$ abbia qualche operazione in comune con $\mathfrak{d}$.

Invero, se $\frac{\mathfrak{Q}}{H}$ è un gruppo, il complesso $G_{1}$, formato con tutte le operazioni di $G$ corrispondenti a $\frac{\mathfrak{Q}}{H}$, è pure un gruppo (11.*68); e poichè $\mathfrak{A}$ è contenuto in $G_{1}$, dovrà essere:

$$
G_{\mathfrak{i}}=\left(\mathfrak{a}, \mathfrak{a}^{-1}\right)=G .
$$

La condizione è poi manifestamente sufficiente. 
Se invece vi è qualche quasi-gruppo $\Delta \cdot g^{\prime}$ che non ha operazioni in comune con $\mathfrak{A}$, il complesso $\frac{\mathfrak{A}}{H}$ è uno pseudogruppo semplice o composto. E precisa. mente: se $\mathfrak{A}$ e $\Delta$ hanno operazioni comuni, $\frac{\mathfrak{Q}}{H}$ è uno psendogruppo composto, altrimenti, $\frac{\mathfrak{Q}}{H}$ è semplice.

78. TeOnema. Affinchè $\frac{\mathfrak{Q}}{H}$ sia abeliano occorye e basta che $\mathrm{H}$ contenga il commutatore $\mathrm{G}_{\mathrm{e}} d i \mathrm{G}=\left(\mathfrak{Q}, \mathfrak{Q}^{-1}\right)$.

Si osservi anzitutto che se $\frac{\mathfrak{Q}}{H}$ è abeliano, tale è anche $\frac{G}{H}$ e reciprocamente.

Nell' ipotesi che $\frac{\mathfrak{A}}{H}$ sia abeliano, ad una commutatrice di $G$ corrisponde in $\frac{G}{H}$ l' identità, onde $G_{0}$ appartiene ad $H$.

Inversamente, se $G_{c}$ appartiene ad $H, \Delta$ contiene $G_{c}\left(\right.$ n. $\left.{ }^{i} 52,58\right)$, e perciò ogni commutatrice di $\frac{G}{H}$ è eguale all' unità.

Ciò prova che $\frac{G}{H}$ e $\frac{\mathfrak{Q}}{H}$ sono abeliani.

CAPITOLO IV.

\section{LE SERIE DI COMPOSIZIONE}

\section{A) I complessi invarianti.}

79. Se un dato complesso $\mathfrak{A}$ è invariante per le operazioni di un complesso $\mathfrak{G}$, il complesso $\mathfrak{A}^{-1}$, formuto con le inverse delle operazioni di $\mathfrak{A}$, $\dot{e}$ pure invariante per $\mathfrak{G}$.

Da ciò consegue l'invarianza per $\widetilde{G}$ dei complessi (a) e $\left(\mathfrak{A}, \mathfrak{A}^{-1}\right)$ generati dalle operazioni di $\mathfrak{a}$ e di $\mathfrak{Q}^{-1}$.

Inoltre, per cose note $\left(1{ }^{\circ}{ }^{\circ} 51\right)$, i complessi $(\mathfrak{d})$ e $\left(\mathfrak{A}, \mathfrak{A}^{-1}\right)$ sono invarianti anche per il gruppo $\left(\widetilde{G}, \mathfrak{G}^{-t}\right)$. 
Consideriamo ora i gruppi chiusi $\left\{\mathfrak{A}, \mathfrak{A}^{-1}\right\},\left\{\mathfrak{G}, \mathfrak{G}^{-1}\right\}$.

Una operazione qualunque $\tau=\prod_{r=1}^{\infty} t$, di $\left\{\widetilde{\sigma}, \mathcal{G}^{-1}\right\}$ trasforma l'operazione generica $a$ di $\left(\mathfrak{A}, \mathfrak{Q}^{-1}\right)$ in una operazione di $\left\{\mathfrak{A}, \mathfrak{A}^{-1}\right\}$ perchè la trasformata $\tau^{-1} \cdot a \cdot \tau$ è il limite della successione:

$$
t_{1}^{-1} \cdot a \cdot t_{1}, \quad t_{2}^{-1} \cdot t_{1}^{-1} \cdot a \cdot t_{1} \cdot t_{2}, \cdots
$$

i cui termini, per quanto $\mathrm{s}^{\prime}$ è detto superiormente, appartengono ad $\left(\mathfrak{A}, \mathfrak{Q}^{-1}\right)$.

Sia poi $k=\prod_{r=1}^{\infty} a$, un prodotto infinito convergente formato con operazioni di $\left(\mathfrak{Q}, \mathfrak{Q}^{-1}\right)$. La trasformata di $k$ mediante $\tau$ appartiene ad $\left\{\mathfrak{Q}, \mathfrak{Q}^{-1}\right\}$ perchè i fattori del prodotto:

$$
\prod_{r=1}^{\infty} \tau^{-1} \cdot a_{r} \cdot \tau=\tau^{-1} \cdot k \cdot \tau
$$

appartengono, come già s'è visto, al gruppo chiuso $\left\{\mathfrak{A}, \mathfrak{A}^{-1}\right\}$.

Possiamo dunque affermare che: se $\mathfrak{A}$ è invariante per. $\mathfrak{G}$, il gruppo $\left\{\mathfrak{A}, \mathfrak{A}^{-1}\right\}$ e invariante per $\left\{\mathfrak{\sigma}, \widetilde{\sigma}^{-1}\right\}$.

OsservaZione. I complessi $(\mathfrak{A})$, $(\mathfrak{Q}, \mathfrak{Q}-1)$, invarianti per $\left(\mathfrak{G}, \mathfrak{T}^{-1}\right)$, non sono, in generale, invarianti per $\left\{\mathfrak{T}, \mathfrak{G}^{-1}\right\}$.

Invero, la trasformata $\tau^{-1} \cdot a \cdot \tau$, essendo il valore di un prodotto infinito di operazioni di $\left(\mathfrak{A}, \mathfrak{A}^{-1}\right)$, non sempre appartiene ad $(\mathfrak{Q})$ e ad $\left(\mathfrak{A}, \mathfrak{A}^{-1}\right)$.

80. Dalle precedenti proposizioni si deduce immediatamente che: I complessi :

$$
\left|(\mathfrak{A}),\left(\mathfrak{G}, \mathfrak{G}^{-1}\right)\right|, \quad\left|\left(\mathfrak{A}, \mathfrak{A}^{-1}\right),\left(\mathfrak{G}, \mathfrak{G}^{-1}\right)\right|, \quad \mid\left\{\mathfrak{A}, \mathfrak{A}-1\left|,\left(\mathfrak{G}, \mathfrak{G}^{-1}\right)\right|\right.
$$

sono invarianti per $\left(\mathfrak{G}, \mathfrak{G}^{-1}\right)$ ed il gruppo:

$$
\left|\left\{\mathfrak{a}, \mathfrak{A}^{-1}\right\},\left\{\mathfrak{G}, \mathfrak{G}^{-1}\right\}\right|
$$

è invariante per $\left\{\widetilde{\sigma}, \widetilde{\sigma}^{-1}\right\}$.

81. Teonema I. Se ciascuno dei complessi $\mathfrak{A}, \mathfrak{G}$ è invariante per l'altro il prodotto a.t è commulabile a meno di una operazione del gruppo:

$$
D=\left|\left(\mathfrak{Q}, \mathfrak{Q}^{-1}\right),\left(\mathfrak{G}, \mathfrak{G}^{-1}\right)\right|
$$

Per le ipotesi fatte la commutatrice di $a$ e $t$ :

$$
a^{-1} \cdot t^{-1} \cdot a \cdot t=a^{-1} \cdot\left(t^{-1} \cdot a \cdot t\right)=\left(a^{-1} \cdot t^{-1} \cdot a\right) \cdot t
$$


appartiene ai gruppi $\left(\mathfrak{A}, \mathfrak{A}^{-1}\right),\left(\mathcal{G}, \mathfrak{G}^{-1}\right)$ e perciò appartiene a $D$. Posto quindi $d=a^{-1} \cdot t^{-1} \cdot a \cdot t$, si ha:

$$
a \cdot t=t \cdot a \cdot d
$$

82. Teorema II. Se $\mathfrak{A}$ è invariante per $\mathfrak{G}, i$ complessi:

$$
\frac{\mathfrak{a} \cdot \mathscr{\mathfrak { C }}}{\left(\mathfrak{a}, \mathfrak{a}^{-1}\right)}, \quad \frac{\mathfrak{G}}{\left|\left(\mathfrak{a}, \mathfrak{a}^{-1}\right),\left(\mathfrak{G}, \mathfrak{G}^{-1}\right)\right|}
$$

sono isomorfit oloedricamenle.

Poniamo, per semplicità di scrittura:

$A=\left(\mathfrak{Q}, \mathfrak{Q}^{-1}\right), \quad T=\left(\mathfrak{G}, \mathfrak{G}^{-1}\right), \quad \mathfrak{L}=\mathfrak{A} \cdot \mathcal{G}, \quad L=\left(\mathfrak{L}, \mathfrak{L}^{-1}\right), \quad D=|A, T|$

Se nella eguaglianza:

$$
L=A \cdot T
$$

sostituiamo al gruppo $T$ una sua decomposizione:

$$
T=\Sigma(D \cdot \tau)
$$

rispetto a $D$, otteniamo:

$$
L=A \cdot \Sigma(D \cdot \tau)=\Sigma(A \cdot D \cdot \tau)=\Sigma(A \cdot \tau) .
$$

Dalle $(o)$ e $(\omega)$ discende che i gruppi complementari $\frac{L}{A}, \frac{T}{D}$ hanno il medesimo ordine e che fra essi si può stabilire un isomorfismo oloedrico ponendo in corrispondenza quelle loro operazioni che corrispondono rispettivamente ai quasi-gruppi $A \cdot \tau$ e $D \cdot \tau$.

Consideriamo ora una operazione $t=d \cdot \tau$ appartenente a $\boldsymbol{G}$ ed al quasigruppo $D \cdot \tau$ di $(o)$. Il prodotto:

$$
a \cdot t=a \cdot d \cdot \tau,
$$

di una qualsiasi operazione $a$ di $\mathfrak{Q}$ per $t$, appartiene al complesso $\mathfrak{L}$ ed al quasigruppo $A \cdot \tau$ di $(\omega)$.

Inversamente, se l'operazione $a \cdot t$ di $\mathcal{L}$ appartiene al quasi-gruppo $A \cdot \tau$, si ha:

$$
a \cdot t=\alpha \cdot \tau, \quad a^{-1} \cdot \alpha=t \cdot \tau^{-1}=d, \quad d \cdot \tau=t,
$$

e cioè nel quasi-gruppo $D \cdot \tau$ figura l'operazione $t$ di $\mathfrak{G}$.

Da ciỏ consegue che le operazioni dei complessi $\frac{\mathfrak{L}}{A}$ e $\frac{\mathfrak{G}}{D}$ si corrispondono 
nell' isomorfismo che intercede fra $\frac{A}{L}$ e $\frac{T}{D}$, e che percio $\frac{\mathscr{L}}{A}$ e $\frac{\mathscr{G}}{D}$ sono oloedricamente isomorfi.

83. Sia $G_{m}$ un sottogruppo invariante massimo di un gruppo $G$, e sia $\Gamma$ un altro sottogruppo invariante di $G$ non contenuto in $G_{m}$.

Dal teoremil precedente, applicato ai complessi $\mathfrak{A}=G_{m}, \widetilde{G}=\Gamma$, si deduce che i gruppi $\frac{G}{G_{m}}, \frac{\mathrm{J}}{\left|\Gamma, G_{m}\right|}$ sono isomorfi oloedricamente.

Inoltre, poichè $G_{m}$ è massimo in $G$, il gruppo $\frac{\Gamma}{\left|\Gamma, G_{m}\right|}$ non può avere sottogruppi invarianti all'infuori della identità, e quindi $\left|\Gamma, G_{m}\right|$ è massimo in $\Gamma$.

\section{B) Le serie di composizione.}

84. Dato un gruppo o pseudogruppo $\mathfrak{C}$, diremo che esso ammette una serie di composizione quando esiste una successione di complessi :

$(\alpha)$

$$
\mathfrak{C}, \quad \mathfrak{C}_{2}, \quad \mathfrak{C}_{3}, \ldots, \mathfrak{C}_{n}, \ldots
$$

tale che :

a) ciascun $\mathfrak{C}_{n}$ sia della stessa specie di $\mathfrak{C}$ e sia invariante massimo nel precedente;

b) presa una operazione qualsiasi $c$ di $\mathfrak{C}$ (esclusa l' identità se esiste in $\mathfrak{C}$ ) esista un complesso $\mathcal{C}_{n}$ tale che esso ed i seguenti non contengano $c$.

85. I complessi complementari :

$$
\frac{\mathfrak{C}}{\left(\mathfrak{C}_{2}, \mathfrak{C}_{2}^{-1}\right)}, \frac{\mathfrak{C}_{2}}{\left(\mathfrak{C}_{3}, \mathfrak{C}_{3}^{-1}\right)}, \ldots, \frac{\mathfrak{C}_{n}}{\left(\mathfrak{C}_{n+1}^{-}, \mathfrak{C}_{n+1}^{-1}\right)}, \ldots
$$

verranno chiamati complessi fattoriali ed i loro ordini si diranno fattori di composizione.

Se $\mathcal{C}$ è un gruppo, i fattori di composizione coincidono con gli indici di $\mathfrak{C}_{2}$ in $\mathfrak{C}, \mathfrak{C}_{3}$ in $\mathfrak{C}_{2}$, ecc.

86. Se in $(\alpha)$ esistono dei complessi eguali ad 1, la serie può arrestarsi al primo di essi : $\mathfrak{C}_{r}$, e si puó scrivere:

$$
\mathfrak{C}, \mathfrak{C}_{2}, \ldots, \mathfrak{e}_{r_{-1}}, 1
$$

In tal caso si dirà che $\mathcal{C}$ ammette una serie funila di composizione. 
Uno pseudogruppo non puỏ ammettere una serie finita perchè ciascun complesso di $(\alpha)$ è uno pseudogruppo e, come tale, non puó ridursi alla identitá.

87. Per un complesso $\mathfrak{C}$ di infinite operazioni, a differenza di quanto avviene per i gruppi finiti, non è sempre possibile di costruire delle serie di composizione. Questa impossibilità si presenta quando per il complesso $\mathfrak{C}$ non si puó soddisfare ad una delle condizioni a) e b) del n..$^{\circ} 84$.

Ad esempio, $i$ complessi costruiti al $n .^{\circ} 55$, non possedendo complessi invarianti massimi, non soddisfano alla condizione $a$ ) e perciò non ammettono serie di composizione.

In altri casi può invece essere soddisfatta la a) ma non la b) : ciò si verifica per il gruppo costruito nel seguente:

EsempIo. Sia $G$ il gruppo dell' Es. I, n." 55, e sia $h$ una operazione a periodo infinito o senza periodo, commutabile con le operazioni di $G$.

Con il gruppo $G$ e con l'operazione $h$ formiamo il nuovo gruppo abeliano:

$$
H=G+G \cdot h+\ldots .+G \cdot h^{n}+\ldots .+G \cdot h^{-1}+\ldots .+G \cdot h^{-n}+\ldots . .
$$

Indichiamo con $H_{m}$ un sottogruppo invariante massimo di $H$ e con $G_{1}$ il gruppo comune a $G$ e ad $H_{m}$.

Se nella decomposizione:

$$
H_{m}=\Sigma\left(G_{1} \cdot k\right)
$$

di $H_{m}$ rispetto a $G_{1}$, sostituiamo a $G_{1}$ un sottogruppo $G_{2}$ di $G$ contenente $G_{1}\left(\right.$ n. $\left.^{\circ} 55\right)$, otteniamo il gruppo:

$$
H_{m_{1}}=\Sigma\left(G_{2} \cdot k\right)
$$

che contiene $H_{m=}$ ed è invariante in $H$.

Il gruppo $H_{m}$ deve dunque, per essere massimo, contenere tutto $G$. Inoltre, poichè $H$ è abeliano, il gruppo $H_{m}$ dovrà avere indice primo $p$ in $H\left(\mathrm{n} .^{\circ} 56\right)$ e quindi dovrà presentarsi sotto la forma:

(o) $H_{m}=G+G \cdot h^{p}+\ldots+G \cdot h^{r p}+\ldots . G \cdot G \cdot h^{-p}+\ldots+G \cdot h^{-r p}+\ldots .$.

Dalla (o) si deduce, ponendo $h^{p}=l$, che il gruppo $H_{m}$ è dello stesso tipo di $H$ e quindi che un sottogruppo massimo di $H_{m}$ deve avere una forma analoga alla $(o)$. E pertanto possibile di costruire per $H$ delle successioni di gruppi contenenti $G$ e ciascuno dei quali è invariante massimo nel precedente.

Queste successioni, soddisfacendo alla condizione $a$ ) ma non alla $b$ ) del $n .^{\circ} 84$, non costituiscono serie di composizione di $H$. 
88. Siano $\mathfrak{C}$ e $\mathfrak{d}$ due complessi isomorfi oloedricamente, e sia:

$$
\mathfrak{C}, \quad \mathfrak{C}_{2}, \ldots, \mathfrak{C}_{n}, \ldots
$$

una serie di composizione di $\mathfrak{C}$.

La successione :

$$
\mathfrak{A K}, \quad \mathfrak{H}_{2}, \ldots, \quad \mathfrak{A K}_{n}, \ldots .
$$

ottenuta sostituendo in $(\alpha)$ ad ogui $\mathfrak{C}_{n}$ il corrispondente $\mathfrak{H}_{n}$ di $\mathfrak{H}$, è una serie di composizione di $\mathfrak{d}$,

Invero, ciascun $\mathfrak{d}_{n}$ è invariante massimo nel precedente $\mathfrak{C K}_{n-1}\left(\mathrm{n} .^{\circ} 62\right)$, e una operazione $k$, scelta ad arbitrio in $\mathfrak{H}$, appartiene solo a quei primi $r$ complessi di $(\beta)$ che corrispondono agli $r$ complessi di $(\alpha)$ contenenti l'operazione $c \cos$.

89. Consideriamo ora un gruppo $C$ isomorfo meriedricamente ad un altro gruppo $K$, e indichiamo con $H$ quel sottogruppo di $C$ che corrisponde alla identità in $K$.

Affinché esista una serie di composizione di $\mathrm{C}$ contenente $\mathrm{H}$, occorve $e$ basta che $\mathrm{H} e \mathrm{~K}$ abbiano ciascuno una serie di composizione e che la serie di $\mathrm{K}$ sia finila.

Se

$$
C, \quad C_{2}, \ldots, \quad C_{r}, \quad H, \quad H_{2}, \ldots
$$

è una serie di $C$ contenente $H$, la successione:

$$
K, \quad K_{2}, \ldots, \quad K_{r}, \quad 1,
$$

formata con i sottogruppi di $K$ corrispondenti a $C, C_{2}, \ldots$, è una serie finita di composizione per $K$.

Inoltre, è evidente che, per l'esistenza di $(\alpha)$, il gruppo $H$ deve possedere una serie di composizione.

Inversamente, ammesso l'esistenza di $(\beta)$ e di una serie per $H$, nella successione corrispondente a $(\beta)$ :

$$
C, \quad C_{2}, \ldots, \quad C_{2}, \quad H
$$

ciascun gruppo è invariante massimo nel precedente, e perció, completando la $\left(\alpha^{\prime}\right)$ con la serie di $H$, si ottiene una serie per $C$.

Osservazione I. Volendo verificare se per un gruppo $C$ esiste una serie di composizione contenente un suo sottogruppo invariante $H$, basterà porre $K=\frac{C}{H}$ e riferirsi al teorema ora dimostrato. 
Osservazione II. Questo teorema cade generalmente in difetto se, in luogo del gruppo $C$, si considera uno pseudogruppo $\mathfrak{C}$.

Prendiamo, per esempio, i complessi :

$$
C^{\prime \prime}=\left[1, g, \ldots, g^{*}, \ldots .,\right], \quad K=[1, k] \quad\left(k^{2}=1\right)
$$

isomorfi meriedricamente secondo le corrispondenze:

$$
H^{\prime \prime}=\left[1, g^{2}, \ldots ., g^{2 n}, \ldots .\right] \text { cos } 1,\left[g, g^{3}, \ldots, g^{2 n+1}, \ldots .\right] \cos k .
$$

Poichè $H^{\prime \prime}$ e $K$ ammettono rispettivamente le seguenti serie di composizione :

$$
\begin{gathered}
H^{\prime \prime}, \quad\left[1, g^{4}, \ldots ., g^{2 n}, \ldots .\right], \quad\left[1, g^{6}, \ldots, g^{2 n}, \ldots .\right], \ldots ., \\
K, 1 ;
\end{gathered}
$$

le condizioni del precedente teorema sono soddisfatte; nonostante ció il complesso $H^{\prime \prime}$ non figura in nessuna serie di composizione di $C^{\prime \prime}$.

Invero, un complesso $C_{1}^{\prime \prime}$ di $C^{\prime \prime}$ contenente $H^{\prime \prime}$ è necessariamente del tipo:

$$
C_{1}^{\prime \prime}=\left[1, g^{2}, g^{4}, \ldots ., g^{2 n}, g^{2 n+1}, g^{2 n+2}, \ldots .\right],
$$

e come tale non ammette $H^{\prime \prime}$ per sottopseudogruppo invariante massimo.

90. Sia

$$
G=\left[1, g, \ldots ., g^{r}, \ldots ; g^{-t}, \ldots ., g^{-r}, \ldots .\right]
$$

il gruppo generato da una operazione $g$ senza periodo o con periodo infinito. Poichè $G$ è abeliano, un suo qualsiasi sottogruppo:

$$
G_{p_{1}}=\left[1, g^{p_{1}}, \ldots ., g^{n p_{1}}, \ldots . . ; g^{-p_{1}}, \ldots . ., g^{-n p_{1}}, \ldots .\right],
$$

avente indice primo $p_{1}$, è invariante massimo in $G\left(\mathbf{n}^{\circ} 56\right)$.

Il gruppo $G_{p_{1}}$, essendo generato dalla operazione $g^{p_{1}}$, è della medesima specie di $G$, e perciò un suo qualsiasi sottogruppo $G_{p_{2}}$, avente indice primo $p_{2}$, sarà massimo in $G_{p_{1}}$.

Continuando in tal guisa si vede agevolmente che una successione di gruppi :

$$
G, \quad G_{p_{1}}, \quad G_{p_{2}}, \ldots, \quad G_{p_{p}}, \ldots .,
$$

ciascuno dei quali ha indice primo nel precedente, costituisce una serie di composizione di $G$.

Poichè la scelta della successione dei fattori di composizione di $(\alpha)$ :

$$
p_{1}, \quad p_{2}, \ldots ., \quad p_{\ell}, \ldots .
$$


è in nostro arbitrio, l' esempio ora portato ci mostra come un gruppo infinito possa avere infinite serie di composizione, e come per esso non sia generalmente valido il noto teorema di JoRDan-HöLDER che assicura l' invarianza dei fattori di composizione e l'isomorfismo dei gruppi fattoriali in due diverse serie di un gruppo finito.

91. Vogliamo ora far vedere che questo teorema puo cadero in difetto soltanto per quei complessi che hanno serje formate con infiniti termini od, in altre parole, vogliamo dimostrare il seguente:

Teorema. Sia G un gruppo infinito avente una serie finita di composizione :

$$
G, \quad G_{2}, \ldots, \quad G_{m-1}, \quad 1 .
$$

Una qualsiasi altra successione di gruppi :

$$
G, \quad H_{2}, \ldots, \quad H_{m-1}, \quad H_{m}, \ldots .
$$

tale che ciascuno di essi sia invariante massimo nel precedenle, è una serie di composizione di $\mathrm{G}$ formata di $\mathrm{m}$ termini (cioè $\mathrm{H}_{\mathrm{m}}=1$ ), ed i.gruppi fattoriali di $(\alpha)$ sono isomorfi oloedricamente ai gruppi fattoriali di $(\beta)$.

Il teorema è evidente per quei gruppi che hanno serie formate con due soli termini.

Supponiamo ora che il teorema sia dimostrato per tutti quei gruppi che ammettono una serie di composizione formata con $m-1$ termini, e sotto tale ipotesi, dimostriamolo per un gruppo $G$ qualunque che abbia una serie di $m$ termini.

Anzitutto osserviamo che il gruppo $H_{2}$ può supporsi diverso da $G_{2}$ perchè, in caso contrario, avendo $G_{2}$ una serie di $m-1$ termini, il teorema sarebbe già dimostrato.

Costruiamo poi i gruppi :

$$
\begin{aligned}
& C_{1}=\left|H_{2}, G_{2}\right|, \\
& C_{2}=\left|C_{1}, G_{3}\right|=\left|H_{2}, G_{2}, G_{3}\right|, \\
& \ldots \ldots \ldots \ldots \ldots \ldots \ldots \ldots \ldots, G_{m-1} \mid .
\end{aligned}
$$

Il gruppo $C_{1}$ è invariante massimo in $G_{2}$ e $H_{2}$, perchè questi sono tali in $G\left(\mathrm{n}^{\circ}{ }^{8} 83\right)$, il gruppo $C_{2}$ è, per la medesima ragione, massimo in $C_{1}$ e $G_{3}$, ed il gruppo generico $C_{r,}$, ammesso che $C_{r-1}$ sia massimo in $C_{r-2}, G_{r}$, risulta invariante massimo in $C_{r-1}$ e $G_{r+1}$. 
Possiamo dunque affermare che ciascum $C_{r}$ è invariante massimo nel precedente $C_{r-1}$.

Inoltre, affinchè un gruppo $C_{s}$ si riduca alla sola operazione identica è necessario che:

$$
G_{s+1}=G_{m-1}
$$

onde l'unico $C_{s}$ eguale ad 1 è il gruppo ultimo $C_{n-2}$.

Da ciò consegue che le due successioni :

$$
\begin{array}{llllll}
G, & G_{2}, & C_{1}, & C_{2}, \ldots ., & C_{m-3}, & 1, \\
G, & H_{2}, & C_{1}, & C_{2}, \ldots, & C_{m-3}, & 1
\end{array}
$$

costituiscono due serie di composizione di $G$.

I gruppi fattoriali di queste serie sono isomorfi oloedricamente perchè (11. 83):

$$
\frac{G}{G_{2}} \cos \frac{H_{2}}{C_{1}}, \frac{G}{H_{2}} \cos \frac{G_{2}}{C_{1}} .
$$

Dalla $(b)$ si deduce che il gruppo $H_{2}$ possiede una serie di $m-1$ termini, e perciò (essendo il teorema vero per quei gruppi che hamno serie di $m-1$ termini) che il gruppo $H_{m}$ deve ridursi alla jdentità.

La $(\beta)$ rappresenta quindi una serie di $G$ formata con $m$ gruppi.

Per le gin note ipotesi fatte circa la validita del teorema, i gruppi fattoriali di $(\alpha)$ e di $(\beta)$ sono rispettivamente isomorfi a quelli di $(a)$ e di $(b)$, e poichè, come abbiamo visto, i gruppi fattoriali di $(a)$ e di $(b)$ sono fra loro isomorfi, saranno pure tali i gruppi di $(\alpha)$ e di $(\beta)$.

In virtù di questi risultati e per il principio d'induzione, il teorema enunciato può ritenersi vero in generale.

92. Diremo che un gruppo o pseudogruppo e è risolubile se esiste una serie di composizione di $\mathcal{C}$ i cui fattori sono numeri primi.

EsempIO I. Il gruppo $G$ costruito al $110^{\circ} 90$ è risolubile.

Esempro II. Lo pseudogruppo:

$$
G^{\prime \prime}=\left[1, g, g^{z}, \ldots, g^{r}, \ldots .\right]
$$

è risolubile poichè ammette la serie :

$$
G^{\prime \prime}, \quad\left[1, g^{2}, \ldots, g^{r}, \ldots .\right], \quad\left[1, g^{3}, \ldots, g^{\prime}, \ldots . ., \ldots\right.
$$

i cui fattori sono tutti eguali ad 1. 
93. Teorema I. Un complesso ubeliano $\mathfrak{C}$, avente una serie di composizione, è risolubile.

Se $\mathfrak{C}$ è un gruppo, il teorema discende immediatamente dalla proposizione del $n .^{\circ} 56$.

Supponiamo invece che $\mathcal{C}$ sia uno pseudogruppo semplice o composto e indichiamo con $\mathfrak{C}_{2}$ un suo sottopseudogruppo invariante massimo.

Sia $c$ una operazione di $\mathfrak{C}$ non appartenente a $\mathfrak{C}_{2} \in$ senza inversa in $\mathfrak{C}$. Una sua qualsiasi potenza $c^{r}$, con $r \geq 2$, deve appartenere a $\mathfrak{C}_{2}$ perchè, se ciò non fosse, lo pseudogruppo:

$$
\left(\mathfrak{e}_{2}, c^{*}\right)
$$

conterrebbe $\mathfrak{C}_{2}$ e non coinciderebbe con $\mathfrak{C}$.

Da ció consegue che il gruppo:

$$
G=\left(\mathfrak{C}_{;}, \mathfrak{e}_{2}^{-1}\right),
$$

contenendo le operazioni $c^{r+1}, c^{-r}$, contiene anche il loro prodotto $c=c^{r+1} \cdot c^{-r}$.

Si puó dunque affermare che nel gruppo $G$ figura qualsiasi operazione di $\mathfrak{C}$ senza inversa in $\mathfrak{C}$.

Sia poi $k$ una operazione di $\mathfrak{C}$ con l'inversa in $\mathfrak{C}$. Il prodotto $k \cdot c$, non avendo l' inversa in $\mathfrak{C}$, appartiene al gruppo $G$, e perció in questo gruppo figura anche l'operazione $k=k \cdot c \cdot c^{-1}$.

$\mathrm{Nel}$ gruppo $G$ è dunque compreso tutto il complesso $\mathfrak{C}$, e poichè $\mathfrak{C}_{2}$ è contenuto in $\mathfrak{C}$, dovrà aversi :

$$
\left(\mathfrak{C}_{2}, \mathfrak{C}_{2}^{-1}\right)=\left(\mathfrak{e}, \mathfrak{e}^{-1}\right)
$$

In modo analogo si prova, essendo:

$$
\mathfrak{e}, \mathfrak{C}_{2}, \mathfrak{C}_{3}, \ldots, \mathfrak{C}_{n}, \ldots .
$$

la serie di composizione di $\mathfrak{C}$, che :

$$
\left(\mathfrak{C}_{n}, \mathfrak{C}_{n}^{-1}\right)=\left(\mathfrak{C}_{n-1}, \mathfrak{C}_{n-1}^{-1}\right),
$$

cioè che i fattori di composizione di $(\alpha)$ sonn tutti eguali ad 1.

94. Teorema II. Se un gruppo Gè risolubile, è puve risolubile qualunque suo sottogruppo $\mathrm{H}$.

Sia

$$
G, \quad G_{2}, \quad G_{3}, \ldots, \quad G_{n}, \ldots
$$


una serie di composizione di $G$ e siano:

$$
p_{1}, \quad p_{2}, \ldots, \quad p_{n-1}, \cdots
$$

i fattori di composizione che, per ipotesi, sono tutti numeri primi.

Con il gruppo dato $H$ e con il primo gruppo $G_{i}$ di $(\alpha)$ che non contiene per intero $H$ si formi il nuovo gruppo:

$$
K=G_{i} \cdot H .
$$

Fra l'indice $q$ di $K$ in $G_{i-1}$ e l'indice $r$ di $G_{i}$ in $K$ passa evidentemente la relazione:

$$
p_{i-1}=q \cdot 1 \cdot
$$

la quale, per essere $p_{i-1}$ primo, è soddisfatta soltanto da $q=1, r=p_{i-1}$. Ció prova che il gruppo $K$ coincide con $G_{i-1}$.

Inoltre, essendo $\frac{G_{i-1}}{G_{i}}$ isomorfo oloedricamente a $\frac{H}{\left|G_{i}, H\right|}$ (Teor. n.. 82), il gruppo $H_{1}=\left|G_{i}, H\right|$ ha indice primo eguale a $p_{i-1}$ in $H$ e perciò $H_{1}$ è invariante massimo in $H$.

In modo del tutto analogo si puó mostrare l'esistenza di un sottogruppo $H_{2}$ invariante massimo di $H_{1}$ ed avente indice primo, e cosi continuando si viene a costruire una serie di composizione di $H$ i cui fattori sono tutti numeri primi. Il gruppo $H$ è dunque risolubile.

95. Dato un gruppo o pseudogruppo $\mathfrak{C}$, diremo che esso ammette una serie principale di composizione quando esiste una successione di complessi :

$$
\mathfrak{C}, \mathfrak{C}_{2}, \mathfrak{C}_{3}, \ldots, \mathfrak{C}_{n}, \ldots
$$

soddisfacente alla condizione b) del $n .^{\circ} 84$ e tale che:

a) ciascuu $\mathfrak{C}_{n}$ sia della stessa specie di $\mathfrak{C}$ e sia invariante in $\mathfrak{C}$;

b) non esista alcun complesso $\mathfrak{D}$ di $\mathfrak{C}_{n-1}$ invariante per $\mathfrak{C}$ e contenente $\mathfrak{C}_{n}$.

96. Come già abbiamo fatto per le serie ordinarie di composizione, distingueremo le serie principali in finite ed infinite, e chiameremo complessi fattoriali principali i complessi :

$$
\frac{\mathfrak{C}}{\left(\mathcal{C}_{2}, \mathfrak{C}_{2}^{-1}\right)}, \frac{\mathfrak{C}_{2}}{\left(\mathfrak{C}_{3}, \mathfrak{C}_{3}^{-1}\right)}, \ldots .
$$


Il teorema di JoRDAN-HöLDER, che abbiamo dato per le serie ordinarie, si puó dimostrare in modo del tutto analogo e sotto le medesime ipotesi per le serie principali.

97. Se un gruppo $\mathrm{G}$ ammette la serie principale:

$$
G, \quad G_{2}, \quad G_{3}, \ldots ., \quad G_{n}, \ldots .
$$

e se il gruppo $\frac{\mathrm{G}_{\mathrm{r}}}{\mathrm{G}_{\mathrm{r}+1}}=\mathrm{K}$ possiede una serie ordinaria finila:

$$
K, \quad K_{2}, \ldots, \quad K_{m-1}, \quad 1,
$$

la successione dei gruppi comispondenti di $\mathrm{G}_{\mathrm{r}}$ :

$$
G_{r} ; \quad G_{r}^{(2)}, \ldots, \quad G_{r}^{(m-1)}, \quad G_{r+1}
$$

è tale che due gruppi qualsiasi:

$$
\frac{G_{r}^{(8)}}{G_{r}^{(8+1)}}, \frac{G_{r}^{(t)}}{G_{r}^{(t+1)}}
$$

sono isomoifi oloedricamente.

La dimostrazione di questo teorema, che qui per brevità omettiamo, è del tutto identica alla dimostrazione dell' analoga proposizione relativa ai gruppi finiti.

Se poi tutti i gruppi fattoriali principali di $(\alpha)$ hanno serie ordinarie finite, è possibile, per il Teor. del n. ${ }^{\circ} 89$, costruire una serie ordinaria di composizione di $G$ contenente tutti $\mathrm{j}$ gruppi di $(\alpha)$; ed inversamente, se esiste una tale serie ordinaria, $i$ gruppi fattoriali principali di $(\alpha)$ hanno serie finite.

Da tutto ciò segue che:

Se un gruppo G annelte una serie principale (o), in qualsiasi serie ordinariā di $\mathrm{G}$ contenente tutti $i$ gruppi di $(\alpha)$ :

$$
G ; \quad G_{2}, G_{2}^{(2)}, G_{2}^{(3)}, \ldots ; G_{3}, G_{3}^{(2)} \ldots ; \ldots ; G_{r}, G_{r}^{(2)}, \ldots . G_{r}^{(m)} ; G_{r+1} \ldots
$$

i complessi faltoriali da $\frac{\mathrm{G}_{\mathrm{r}}}{\mathrm{G}_{\mathrm{r}}^{(3)}}$ a $\frac{\mathrm{G}_{\mathrm{r}}^{(\mathrm{m})}}{\mathrm{G}_{\mathrm{r}+1}}$ sono isomorfi oloedricamente. 